\title{
Research advances in myocardial injury caused by COVID-19
}

\author{
Jianjun Chen ${ }^{1 \#}$, Yingfeng $\mathrm{He}^{1 \#}$, Liangliang Zhou ${ }^{1}$, Genhua $\mathrm{Mu}^{1}$, Youlin $\mathrm{Ji}^{2}$ \\ ${ }^{1}$ Department of Critical Care Medicine, ${ }^{2}$ Department of Respiratory and Critical Care Medicine, Yancheng No.1 People's Hospital, Yancheng, China \\ Contributions: (I) Conception and design: Y Ji; (II) Administrative support: G Mu; (III) Provision of study materials or patients: Y He, L Zhou; (IV) \\ Collection and assembly of data: L Zhou; (V) Data analysis and interpretation: Y He; (VI) Manuscript writing: All authors; (VII) Final approval of \\ manuscript: All authors. \\ \#These authors contributed equally to this work. \\ Correspondence to: Dr. Youlin Ji. Department of Respiratory and Critical Care Medicine, Yancheng No.1 People's Hospital, No.66 Renmin South \\ Road, Yandu District, Yancheng, China. Email: chenjianjun13387@163.com.
}

\begin{abstract}
Coronavirus disease 2019 (COVID-19) caused a large-scale infection in China at the end of 2019. The virus, named severe acute respiratory syndrome coronavirus 2 (SARS-CoV-2), has the characteristics of strong transmission capacity, diverse clinical manifestations, long incubation period, strong infection in incubation period, etc. With the increase of the number of cases and the continuous improvement of clinical data, we found that patients with COVID-19 have myocardial injury besides typical respiratory system manifestations. According to published data, we summarized the clinical manifestations of myocardial injury in COVID-19 patients, and discussed the probable injury mechanism, treatment methods and future research directions.
\end{abstract}

Keywords: Coronavirus disease 2019 (COVID-19); myocardial injury; diagnosis; treatment

Submitted Mar 12, 2020. Accepted for publication Jun 12, 2020.

doi: $10.21037 / \mathrm{cdt}-20-349$

View this article at: http://dx.doi.org/10.21037/cdt-20-349

\section{Introduction}

In December 2019, a newly discovered coronavirus, named severe acute respiratory syndrome coronavirus 2 (SARS$\mathrm{CoV}-2$ ), was isolated and identified from respiratory epithelial cells of patients with unexplained pneumonia in Hubei Province of China. Coronavirus disease 2019 (COVID-19) broke out in Wuhan and spread to the whole province and even other provinces, posing a major threat to human life safety and health. As of on May 13, 2020, a total of 4,178,091 confirmed cases of COVID-19 have been reported worldwide, resulting in 294,682 death (1).

SARS-CoV-2, which belongs to the order of nested viruses, coronaviridae, and the genus of coronaviruses $\mathrm{B}$, is a class of enveloped non-segmented single-stranded positive strand RNA viruses with a diameter of 50-200 nm (2), and is the seventh member of the genus of coronaviruses found so far that can infect humans. Among the other six coronaviruses that have been confirmed to infect human beings, four coronaviruses (229E, OC43, NL63 and HKU1) are widespread but only cause common upper respiratory tract infection symptoms, and the other two are SARS$\mathrm{CoV}$ and middle east respiratory syndrome coronavirus (MERS-CoV), which can both cause fatal diseases (3). The whole genome sequencing results show that the sequence consistency of SARS-CoV-2 genome and SARS$\mathrm{CoV}$ genome is $79.5 \%$, and the virus is considered to be a coronavirus related to SARS-CoV (4).

The pathway of SARS-CoV-2 entering host the cell is the same as SARS-CoV, i.e., through angiotensin converting enzyme 2 (ACE2). Current epidemiological data show that SARS-CoV-2 may not be as lethal as SARS$\mathrm{CoV}$ and MERS-CoV. An epidemiological survey that included 44,672 confirmed cases revealed that the crude fatality rate after SARS-CoV-2 infection was $2.3 \%$, and the fatality rate density was $0.015 / 10$ person days. However, the rapid increase in the number of cases and clear evidence of interpersonal transmission show that the virus is more infectious than SARS-CoV and MERS-CoV (5). Frozen electron microscope test showed that SARS-CoV-2's 
affinity with ACE2 is 10-20 times higher than that of SARS-CoV, which also indicated that the virus is highly infectious (6).

COVID-19 mainly involves respiratory tract, and its clinical first manifestations are fever, dry cough, fatigue and wheezing. Severe cases may develop to severe pneumonia, acute respiratory distress syndrome, systemic multiple organ dysfunction, etc. (7). It is worth noting that more and more data showed that COVID-19 not only causes typical respiratory symptoms, but also affects cardiovascular system, especially serious myocardial damage, thus aggravating the disease condition and affecting the prognosis. Based on the research results published so far, we discuss the manifestations, characteristics, laws and effects on prognosis of myocardial injury caused by COVID-19 in this review.

\section{Myocardial injury in COVID-19 patients}

Irrespective of the presence or absence of basic cardiovascular diseases, abnormal elevation of myocardial injury markers widely existed in COVID-19 patients, and was closely related to disease progression and prognosis. Among 41 patients diagnosed as COVID-19 published by Fan et al. (7), 5 patients (12\%) were definitely diagnosed as acute myocardial injury, mainly manifested as highsensitivity cardiac troponin I (hs-cTnI) level rising $(\geq 28 \mathrm{ng} / \mathrm{L})$. One of them was a mild case, accounting for $4 \%$ of all mild cases. Four patients were admitted to intensive-care unit (ICU), accounting for $31 \%$ of the total number of severe patients. In another retrospective study, which included 99 confirmed patients with COVID-19, most patients had elevated myocardial injury markers, specifically, creatine kinase (CK) increased ( $\geq 310 \mathrm{U} / \mathrm{L})$ in $13 \%$ of patients and lactate dehydrogenase $(\mathrm{LDH})$ increased $(\geq 250 \mathrm{U} / \mathrm{L})$ in $76 \%$ of patients. In another single-center retrospective study involving 138 confirmed patients with COVID-19 (5), 10 patients (7.2\%) were diagnosed with acute myocardial injury, of which two were mild cases, accounting for $2 \%$ of the total number of mild cases. Eight cases were severe patients admitted to ICU, accounting for $22 \%$ of the total number of severe patients. Moreover, compared with mild patients, the CK isoenzyme (CK-MB), LDH and hs-c TnI of severe patients were higher, proving that COVID-19 infected myocardial damage was related to the progression of the disease. Among the earliest published death, one confirmed patient was admitted with severe COVID-19 infection-related myocarditis as the main manifestation, with myocardial injury markers as high as 20 times of the normal upper limit, and corresponding electrocardiographic abnormalities (8).

The first multicenter retrospective study included 1,099 COVID-19 patients from 552 hospitals in 31 provinces in China (9). According to the guidelines for adult communityacquired pneumonia issued by the American Chest Association, the study classified patients into severe and non-severe groups. The main composite endpoint events included ICU admission, mechanical ventilation and death. The results revealed that the expression level of myocardial injury markers was higher in severe cases and patients with major composite endpoint events. In an observational study involving 675 patients, 277 patients (41\%) had LDH elevation ( $\geq 250 \mathrm{U} / \mathrm{L}$ ), accounting for $37.2 \%$ of non-severe patients and $58.1 \%$ of severe patients. LDH elevation occurred in $70.5 \%$ of patients with major composite endpoint events, while only $39.0 \%$ of patients without major composite endpoint events. Among 657 patients with CK data recorded, 90 patients $(13.7 \%)$ had CK elevation ( $\geq 200 \mathrm{U} / \mathrm{L}$ ). Compared with patients without major composite endpoint events, patients with major composite endpoint events had a higher proportion of CK elevation $(26.1 \% v s$. $12.8 \%, \mathrm{P}=0.021)$. The above study also confirmed that myocardial injury was closely connected with the severity of the disease and even the prognosis in COVID-19 patients. In addition, in a case of familial aggregation in Shenzhen, China, LDH increased ( $\geq 214 \mathrm{U} / \mathrm{L})$ in three of the five confirmed patients with COVID-19, and the three patients were all over 60 years old, with more severe symptoms after onset (7). The first confirmed case of COVID-19 reported in the United States also had a broad rise in $\mathrm{LDH}$ during hospitalization (10).

\section{Clinical manifestations of myocardial injury caused by COVID-19}

The most common clinical symptoms of patients with COVID-19 were fever (87.9\%) and cough $(67.7 \%)$. Other manifestations included fatigue (38.1\%), cough up phlegm (33.4\%), etc. (11). However, with the increasing number of confirmed cases, it was not uncommon for patients with COVID-19 whose first symptom was the cardiovascular system. Of the 99 confirmed cases reported by Chen et al. (10), 2 cases (2\%) showed chest pain on admission. However, in a multicenter retrospective study in Hubei province that included 137 confirmed patients with COVID-19, 10 patients (7.3\%) showed palpitations as 
the first symptom (12). According to clinical observation, COVID-19 patients with myocardial injury often showed sinus tachycardia, especially nocturnal tachycardia, and the acceleration of heart rate was not commensurate with the increase of body temperature $\left(\geq 10\right.$ times $\left./{ }^{\circ} \mathrm{C}\right)(5)$. These typical clinical manifestations of cardiovascular diseases suggested the possibility of viral infection involving myocardium. Of course, the possibility of pleural involvement or fever causing the above symptoms needs to be ruled out, and whether myocardial injury related to COVID-19 infection can be clearly diagnosed need further verification.

Based on the characteristics of COVID-19 and the current consensus on diagnosis and treatment of myocarditis in China $(2,13)$, the diagnosis of fulminant myocarditis caused by COVID-19 mainly depends on clinical evidence. If clinicians want to correctly and timely diagnose patients with myocarditis, they need to comprehensively consider the clinical manifestations (hemodynamic disorders), laboratory examinations (myocardial injury markers increase), imaging examinations (echocardiography abnormalities) and changes in etiological examination results (13).

\section{Clinical manifestations}

Individual patients varied greatly, ranging from mild chest pain, palpitation and transient electrocardiogram to lifethreatening cardiogenic shock and malignant arrhythmia. A European study revealed that when myocardial damage occurs due to viral infection, $72 \%$ of patients have dyspnea as the initial symptom, $32 \%$ have chest pain, and $18 \%$ have arrhythmia (14). Explosive myocarditis could cause acute left heart failure or cardiogenic shock. Most of the patients showed pulmonary congestion or shock. Some patients experienced syncope and sudden death.

\section{Laboratory examination}

The gradual increase of myocardial injury markers such as troponin, CK and its isoenzymes, and myoglobin indicated that the disease is deteriorating continuously. Persistent absence of peak values might indicate a poor prognosis. The level of B-type natriuretic peptide (BNP) or N-terminal BNP (NT-proBNP) was typically significantly increased, indicating serious cardiac function damage. At the same time, some studies have demonstrated that the inflammatory reaction produced by COVID-19 also has myocardial injury effect, so we should be alert to abnormal increase of inflammatory indexes in patients (15).

\section{Electrocardiogram examination}

It has high sensitivity but low specificity. Patients should be repeatedly examined. If there is a wide range of limb lead, especially the low voltage of the QRS complex in the chest lead, the patient's myocardial injury might be serious. The occurrence of bundle branch block or atrioventricular block usually indicated a poor prognosis. ST-T segment changes were more common in electrocardiogram examination, representing myocardial repolarization abnormalities. Electrocardiogram of some patients might even be similar to acute myocardial infarction. It is sometimes difficult to distinguish the two, and coronary angiography can be performed for exploration (13).

\section{Echocardiography}

Echocardiography is of great value in diagnosis and followup of myocardial injury caused by COVID-19. Patients might have various degrees of diffuse ventricular wall motion reduction, cardiac systolic dysfunction, cardiac chamber enlargement, ventricular septal thickening caused by myocardial edema, and ventricular wall segmental motion abnormalities (16).

\section{Magnetic resonance imaging}

Specific magnetic resonance imaging manifestations of severe myocarditis included myocardial edema, hyperemia, necrosis, fibrosis, etc. It can be used to distinguish acute myocardial ischemia from myocarditis. However, patients with COVID-19 complicated with myocardial injury were generally in poor condition and their respiratory function was inhibited (2). Nuclear magnetic resonance examination has some risks. In addition, the diagnostic value of MRI for subacute phase was controversial (13).

\section{Percutaneous endocardial myocardial biopsy}

Myocardial Biopsy is still the objective standard for diagnosis of myocarditis, which is helpful for clear diagnosis. However, patients with acute myocardial injury often need to take anticoagulant and/or antiplatelet drugs (13). Therefore, we suggest that only patients with a negative echocardiogram and myocardial magnetic resonance examination should consider this examination method. 


\section{Mechanism of myocardial injury induced by COVID-19}

The pathogenesis of COVID-19 infection-related acute myocardial injury is still unclear, and the following three mechanisms may play a significant role.

(I) SARS-CoV-2 can directly infect and invade myocardial cells, causing myocardial cell damage and viral myocarditis. It invades human cells through high affinity binding of $S$ protein with ACE2, which is highly expressed in myocardial tissue and plays an important role in cardiovascular system (17). Although myocardial injury markers and electrocardiographic abnormalities in some patients highly suggested that SARSCoV-2 directly infects myocardium and leads to myocarditis, and SARS-CoV and MERS-CoV both have reports of myocarditis (11), more exact pathological and imaging evidence is still needed to be confirmed.

(II) Hypoxemia, respiratory failure, shock or hypotension induced by pulmonary infection lead to insufficient oxygen supply to myocardium. After infection, the body metabolism is vigorous, the heart burden increases, and the imbalance of oxygen supply and demand causes myocardial damage, especially for patients suffering from chronic cardiovascular diseases such as coronary heart disease and heart failure.

(III) Immune response disorders, cytokine storms may also lead to myocardial damage. Previous studies have confirmed that cytokine storms play a crucial role in the pathogenesis of SARS-CoV and MERS-CoV, and may be related to cardiovascular system complications related to SARS-CoV infection (11). Chen et al. found that during SARS-CoV-2 infection, the increase of proinflammatory factors such as interleukin (IL)- $1 \beta$, interferon- $\gamma$, interferon inducible protein-10 and monocyte chemoattractant protein-1 may lead to the activation of helper T lymphocyte 1 [adaptive T helper 1 (Th1)] (10). Moreover, compared with mild COVID-19 patients, the concentrations of granulocyte colony stimulating factor, interferon inducible protein-10, monocyte chemotactic protein and tumor necrosis factor- $\alpha$ in plasma of severe patients are higher, suggesting that cytokine storms are closely related to the severity of the disease. A study that included 123 patients found that compared with mild cases, severe cases had higher plasma concentrations of IL- 6 and IL-10 and lower CD4+ T and CD8+ T cells (18). Another study that included 40 patients also reached the same conclusion (19). However, in Guan et al.'s research, cytokines related to helper $\mathrm{T}$ lymphocyte 2 [adaptive $\mathrm{T}$ helper 2 (Th2)] that could reduce inflammatory response, such as IL-4, had also increased (9), which was the feedback regulation of the body on excessive inflammatory response or other effects, yet to be revealed by further research. In a word, SARS-CoV-2 infection may lead to an imbalance of Th1 and Th2 cells. The cytokine storm caused by COVID-19 may be one of the important mechanisms of myocardial injury, and may also be part important mechanism of multiple organ failure caused by immune imbalance.

(IV) Vasculitis refers to the infiltration of inflammatory cells in the vascular wall and its surroundings, accompanied by vascular injuries such as cellulose deposition, collagen fibrosis, endothelial cell necrosis, etc. Vasculitis can be broadly divided into primary vasculitis and secondary vasculitis. The heart is the target organ of almost all primary vasculitis (20). According to "Notice on issuing the diagnosis and treatment plan for novel coronavirus (trial 7th edition)" (21), the pathological biopsy of the heart indicates that myocardial blood vessels were characterized by endothelial shedding, intimal inflammation and thrombosis, which indicated that COVID-19 may induce endothelial cell function decline or even endothelial cell death and shedding, accompanied by endothelial and subcutaneous inflammatory cell infiltration, and the body has a high risk of thrombosis. However, the above changes can lead to acute myocardial injury by affecting myocardial blood supply, inducing myocardial inflammatory reaction and increasing coronary blood flow load.

According to the recent autopsy results and clinical experience, we found that the main organs involved in COVID-19 are lung, heart and kidney $(22,23)$. These three organs had similar pathological changes of vasculitis: mononuclear cells, lymphocyte infiltration and intravascular thrombosis in lung tissue; myocardial vessels showed endothelial shedding, endocarditis and thrombosis. The kidney was characterized by protein exudation of glomerular capillary network and interstitial microthrombosis. Although there was no definite report on whether COVID-19 inclusion bodies exist in blood vessels at present, we speculate that SARS$\mathrm{CoV}-2$ may induce generalized vasculitis through direct and indirect actions based on the fact that these three organs are vascular rich tissues and highly express ACE2 in endothelial cells. On the one hand, SARS-CoV-2 attacks blood vessels (mainly endothelium), leading to the shedding of endodermis and secondary thrombosis, destroying the blood supply of endothelium-dependent tissues, leading to the reduction or interruption of energy metabolism of organs, causing vascular inflammation and thus expanding the inflammatory response of tissues. On the other hand, SARS-CoV-2 stimulates 
systemic inflammatory factor storm. Inflammatory factor induces endothelial cell injury, death and shedding by initiating apoptosis pathway. At the same time, inflammatory factors can further activate endothelial cells, and the activated endothelial cells express a large number of adhesion molecules, which mediate the adhesion and invasion of inflammatory cells to vascular walls or perivascular areas (24).

(V) Pathological studies revealed that myocardial cells of patients with COVID-19 had degeneration and necrosis to vary degrees (21). Hypoxemia might be the physiological basis of this pathological change. Oxygen, as an energy substance of myocardium, is utilized to generate adenosine triphosphate (ATP) to supply electrophysiological contraction and relaxation activities of myocardial cells $(22,25)$. The decrease of oxygen content is firstly manifested by the depletion of ATP and the shortage of energy and materials. In order to satisfy the needs of cell metabolism, myocardial cells increase the number of mitochondria in a short time by inducing mitochondrion to divide itself, thus ensuring oxidative phosphorylation of myocardial cells. However, this abnormal mitochondrion division will induce imbalance of mitochondrial structure and function, which are manifested as instability of the mitochondrial genome, reduction of mitochondrial membrane potential and activation of mitochondrial-dependent apoptosis pathway, thus leading to myocardial cell death. Hypoxemia, which is sometimes difficult to correct, will cause acidosis and oxidative stress of myocardial cells. Excessive oxidative stress and intracellular acidosis jointly act on the endogenous apoptosis pathway of cells, thus inducing myocardial cell death. At the same time, cytokine storms induced by novel coronavirus can further aggravate the death of myocardial cells by increasing the content of tumor necrosis factor $\alpha$ and activating exogenous apoptosis pathways. The above pathophysiological process can explain the changes of myocardial cell degeneration and necrosis in the pathological results of patients with COVID-19. Clinically, we observed that $16.7 \%$ of the patients with COVID-19 had arrhythmia, which was manifested as premature beats, suggesting that COVID-19 infection can improve myocardial self-regulation and excitability $(19,26,27)$.

\section{Treatment of myocardial injury caused by COVID-19}

At present, the exact mechanism of myocardial damage caused by COVID-19 infection is not completely clear (23), but through the above basic and clinical studies, it can be clearly confirmed that COVID-19 infection can cause myocardial damage, and is closely related to the progression and prognosis.

From "Diagnosis and clinical management of 2019 novel coronavirus (2019-nCoV) infection: an operational recommendation of Peking Union Medical College Hospital (V2.0)" (28) to "Notice on issuing the diagnosis and treatment plan for novel coronavirus (trial 7th edition)" (21), it is recommended to use lopinavir/ritonavir (LPV/r) for the treatment of COVID-19. Ritonavir can inhibit CYP3A enzyme in the cytochrome $\mathrm{P} 450$ family, thus inhibiting metabolism of lopinavir mediated by CYP3A enzyme to improve the effective concentration duration of the latter. Therefore, when combined with drugs metabolized by liver CYP3A4 and having high first-pass effect, such as amiodarone, rivaroxaban, warfarin, atorvastatin, rosuvastatin, simvastatin, felodipine and nifedipine, attention should be paid to adjusting the drug dosage to avoid serious or even fatal adverse reactions caused by the increase of drug concentration.

Patients should also be actively treated for cardiovascular diseases when given antiviral drugs. The American College of Cardiology (ACC) recently issued a notice on the impact of COVID-19 on the heart, suggesting that patients with COVID-19 complicated with cardiovascular diseases should be actively treated with statins, beta blockers, ACE inhibitors, aspirin and other drugs to protect cardiovascular diseases as appropriate, and that priority should be given to infected patients complicated with chronic cardiovascular diseases (12). But a study by FitzGerald (29) revealed that NSAIDs, such as PGE2, PGD2, and prostacyclin (PGI2) can both promote and restrict infection. The infection of certain immune cells (microglia) with a related coronavirus (not the one that causes COVID-19) activates a proinflammatory response (the inflammation) to combat the pathogen; This implied that NSAIDs drugs may even lead to exacerbation of symptoms in patients with COVID-19. At the same time, if acute myocardial injury occurs during the course of COVID-19, myocardial protection and nutrition drugs should be reasonably applied, such as coenzyme Q10 synthesized by increasing ATP, creatine phosphate sodium, vitamin C, polar solution, deep sea fish oil, etc. In addition, if cardiac muscle injury leads to arrhythmia, corresponding treatment should be carried out in accordance with arrhythmia types and combined with the hemodynamic status of patients. Once there are signs of heart pump failure, life support treatment such as extracorporeal membrane oxygenation should also be given 
as soon as possible. The most important thing is to build the awareness and vigilance of myocardial damage caused by COVID-19 infection, and to strengthen the monitoring of cardiac function and myocardial damage.

Traditional Chinese medicine treatment for patients with severe COVID-19 focuses on detoxifying and eliminating dampness, clearing heat and relieving asthma. Research by experts in traditional Chinese medicine revealed that SARS-CoV-2 combines with moisture in human body and attacks lungs, thus causing chest tightness, expectoration, shortness of breath and other symptoms. The treatment plan should detoxify and eliminate dampness, and promote good circulation of the lung to eliminate dampness. If the patient's circulatory blood flow is obstructed, which leads to stagnation and blood stasis, the treatment method is mainly to remove blood stasis and unblock collateral (30).

\section{Conclusions}

At present, there are few research data on myocardial injury caused by COVID-19, and there is a lack of pathological data of patients with myocardial injury. The pathological anatomy of the first death patient revealed that the myocardial tissue of the patient showed only a small amount of inflammatory infiltration of myocardial interstitial mononuclear cells, and no substantial myocardial damage was found. However, in view of the fact that this patient has no clinical manifestations of myocardial injury during the whole course of onset, based on the pathological results of this patient, it cannot be concluded that COVID-19 infection does not affect myocardium. In the future, pathological examination of myocardial tissue of COVID-19 patients complicated with definite myocardial injury will be still needed to provide evidence of etiology and etiology for the study of myocardial injury. It is expected that there will be more targeted researches on the mechanism, manifestations, laws, susceptible factors, outcomes and prognosis of myocardial injury of COVID-19 in the future, so as to promote and continuously improve targeted treatment programs and improve the prognosis of patients.

\section{Acknowledgments}

Funding: None.

\section{Footnote}

Conflicts of Interest: All authors have completed the
ICMJE uniform disclosure form (available at http://dx.doi. org/10.21037/cdt-20-349). The authors have no conflicts of interest to declare.

Ethical Statement: The authors are accountable for all aspects of the work in ensuring that questions related to the accuracy or integrity of any part of the work are appropriately investigated and resolved.

Open Access Statement: This is an Open Access article distributed in accordance with the Creative Commons Attribution-NonCommercial-NoDerivs 4.0 International License (CC BY-NC-ND 4.0), which permits the noncommercial replication and distribution of the article with the strict proviso that no changes or edits are made and the original work is properly cited (including links to both the formal publication through the relevant DOI and the license). See: https://creativecommons.org/licenses/by-nc-nd/4.0/.

\section{References}

1. Health Emergency Office. The latest situation of novel coronavirus epidemic as of 2400 hours on May 13. 2020. Available online: http://www.nhc.gov.cn/xcs/yqtb/202002/ 18546da875d74445bb537ab014e7a1c6.shtml

2. Peng WY, Gan XQ, Tan Y, et al. Damage mechanisms of 2019-nCoV infectious disease to cardiovascular system and advance in treatment. Chinese Journal of Nosocomiology 2020;30:1177-82.

3. World Health Organization. Novel Coronavirus - China. 2020. Available online: http://www.who.int/csr/don/12january-2020-novel-coronavirus-china/en/

4. Ashour HM, Elkhatib WF, Rahman MM, et al. Insights into the recent 2019 novel coronavirus (SARS-CoV-2) in light of past human coronavirus outbreaks. Pathogens 2020;9:186.

5. Jin $\mathrm{YH}$, Cai L, Cheng ZS, et al. A rapid advice guideline for the diagnosis and treatment of 2019 novel coronavirus (2019-nCoV) infected pneumonia (standard version). Mil Med Res 2020;7:4.

6. Cao Y, Li L, Feng Z, et al. Comparative genetic analysis of the novel coronavirus (2019-nCoV/SARS-CoV-2) receptor ACE2 in different populations. Cell Discov 2020;6:11.

7. Fan HH, Wang LQ, Liu WL, et al. Repurposing of clinically approved drugs for treatment of coronavirus disease 2019 in a 2019-novel coronavirus-related coronavirus model. Chin Med J (Engl) 2020;133:1051-6.

8. Peng YD, Meng K, Guan HQ, et al. Clinical 
characteristics and outcomes of 112 cardiovascular disease patients infected by 2019-nCoV. Zhonghua Xin Xue Guan Bing Za Zhi 2020;48:E004.

9. Guan WJ, Ni ZY, Hu Y, et al. Clinical Characteristics of Coronavirus Disease 2019 in China. N Engl J Med 2020;382:1708-20.

10. Chen L, Liu HG, Liu W, et al. Analysis of clinical features of 29 patients with 2019 novel coronavirus pneumonia. Zhonghua Jie He He Hu Xi Za Zhi 2020;43:E005.

11. Liu J, Zheng X, Tong Q, et al. Overlapping and discrete aspects of the pathology and pathogenesis of the emerging human pathogenic coronaviruses SARS-CoV, MERS-CoV, and 2019-nCoV. J Med Virol 2020;92:491-4.

12. Cascella M, Rajnik M, Cuomo A, et al. Features, evaluation and treatment coronavirus (COVID-19). In: StatPearls. Treasure Island: StatPearls Publishing, 2020.

13. An TF, Wang WK, Zhao N. Influence of BNP, D-dimer, fibrinogen and blood glucose levels on prognosis in patients with acute myocardial infarction. J Intern Med 2017;23:498-500.

14. Hufnagel G, Pankuweit S, Richter A, et al. The European Study of Epidemiology and Treatment of Cardiac Inflammatory Diseases (ESETCID). First epidemiological results. Herz 2000;25:279-85.

15. Lazaros G, Oikonomou E, Tousoulis D. Established and novel treatment options in acute myocarditis, with or without heart failure. Expert Rev Cardiovasc Ther 2017;15:25-34.

16. Felker GM, Boehmer JP, Hruban RH, et al. Echocardiographic findings in fulminant and acute myocarditis. J Am Coll Cardiol 2000;36:227-32.

17. Liu Z, Xiao X, Wei X, et al. Composition and divergence of coronavirus spike proteins and host ACE2 receptors predict potential intermediate hosts of SARS-CoV-2.J Med Virol 2020;92:595-601.

18. Ling $\mathrm{Y}, \mathrm{Xu} \mathrm{SB}$, Lin YX, et al. Persistence and clearance of viral RNA in 2019 novel coronavirus disease rehabilitation patients. Chin Med J (Engl) 2020;133:1039-43.

19. Bertero E, Maack C. Calcium signaling and reactive oxygen species in mitochondria. Circ Res 2018;122:1460-78.

20. Miloslavsky E, Unizony S. The heart in vasculitis. Rheum
Dis Clin North Am 2014;40:11-26.

21. General Office of the National Health and Health Commission, State Administration of Traditional Chinese Medicine Office. Notice on issuing the diagnosis and treatment plan for novel coronavirus (trial 7 th edition). 2020. Available online: http://www.nhc.gov.cn/yzygj/s7653 p/202003/46c9294a7dfe4cef80dc7f5912eb1989.shtml

22. Zhou H, Hu S, Jin Q, et al. Mff-dependent mitochondrial fission contributes to the pathogenesis of cardiac microvasculature ischemia/reperfusion injury via induction of mROS-mediated cardiolipin oxidation and HK2/ VDAC1 disassociation-involved mPTP opening. J Am Heart Assoc 2017;6:e005328.

23. Yan R, Zhang Y, Li Y, et al. Structural basis for the recognition of SARS-CoV-2 by full-length human ACE2. Science 2020;367:1444-8.

24. Chen YD, Li YZ, Liu XH, et al. Potential pathophysiological mechanisms underlying COVID19-induced myocardial injury. Chinese Journal of Pathophysiology 2020;36:573-6.

25. Zhou H, Zhang Y, Hu S, et al. Melatonin protects cardiac microvasculature against ischemia/reperfusion injury via suppression of mitochondrial fission-VDAC1-HK2mPTP-mitophagy axis. J Pineal Res 2017;63:e12413.

26. Huang C, Wang Y, Li X, et al. Clinical features of patients infected with 2019 novel coronavirus in Wuhan, China. Lancet 2020;395:497-506.

27. Wang D, Hu B, Hu C, et al. Clinical characteristics of 138 hospitalized patients with 2019 novel coronavirus-infected pneumonia in Wuhan, China. JAMA 2020;323:1061-9.

28. Working Group of 2019 Novel Coronavirus, Peking Union Medical College Hospital. Diagnosis and clinical management of 2019 novel coronavirus (2019-nCoV) infection: an operational recommendation of Peking Union Medical College Hospital (V2.0). Med J PUMCH 2020. [Epub ahead of print].

29. FitzGerald GA. Misguided drug advice for COVID-19. Science 2020;367:1434.

30. Zou B, Li M, Fan T, et al. Experience Summary and Treatment Proposal of Traditional Chinese Medicine for Severe novel coronavirus (COVID-19). Journal of Traditional Chinese Medicine 2020. [Epub ahead of print].
Cite this article as: Chen J, He Y, Zhou L, Mu G, Ji Y. Research advances in myocardial injury caused by COVID-19. Cardiovasc Diagn Ther 2020;10(4):881-887. doi: 10.21037/cdt20-349 